\title{
Concepção do Fórum P\&R LV: avaliação formativa da aprendizagem
}

\author{
Alexandra Joca Gonçalves - ajg.virtual@ gmail.com - SEDUC/CE \\ Joana Laysa Lima Cunha - laysacunha@ gmail.com - IFCE \\ Gilvandenys Leite Sales - denyssales@ gmail.com - IFCE
}

Resumo. Este artigo apresenta uma aplicação, ao Fórum Perguntas e Respostas do Ambiente Virtual de Aprendizagem Moodle, da metodologia ativa denominada Instrução por Pares, associado ao modelo de avaliação não-linear Learning Vectors (Modelo LV), peculiarmente processual, que faz uso de outras linguagens na intermediação e regulagem da aprendizagem. Esta associação permite uma facilitação das intervenções pedagógicas e motivação aos envolvidos, uma vez que utiliza menções qualitativas associadas a uma linguagem iconográfica. Como resultado tem-se uma concepção computacional formativa de avaliação não-linear, denominado Fórum Perguntas e Respostas LV, apresentando ampla aceitação em um curso de EaD.

Palavras-Chave: ava, avaliação formativa não-linear, aprendizagem por pares, moodle, fórum $\mathrm{p} \& \mathrm{r}$ lv.

\section{Q\&A LV Forum conception: learning formative evaluation}

Abstract. This article presents an application, in the Questions and Answers Forum, from the Learning Management System Moodle, of an active methodology known as Peer Instruction, associated with the non-linear evaluation Learning Vectors Model (LV Model), peculiarly procedural, that uses other languages in the learning intermediation and regulation process. This association can facilitate pedagogical interventions and motivate to those involved, once qualitative terms associated with an iconographic language are used. The result is a computational mechanism based on a nonlinear formative assessment model called Questions and Answers LV Forum, which was implemented in a long distance course with wide acceptance.

Keywords: Ims, nonlinear formative evaluation, peer instruction, moodle, q\&a lv forum.

\section{INTRODUÇÃO}

A intensa expansão das Tecnologias Digitais da Informação e Comunicação (TDIC) tem gerado um grande impacto na educação, sobretudo, como artefatos que suportam e conduzem a Educação a Distância (EaD), esta, compreendida pelo distanciamento físico dos sujeitos envolvidos no processo de ensino e de aprendizagem (MOORE; KEARSLEY, 2007).

O uso destas tem adquirido destaque e reconhecimento, transcendendo distâncias e limitações temporais, inclusive prevalecendo a integração de seus elementos: humano, máquina, e conhecimento, respeitando suas especificidades e favorecendo um processo dinâmico de ensino e aprendizagem, facilitando-o. 
Destaque destas no âmbito educacional que propiciaram a ampla utilização de Ambientes Virtuais facultando à aprendizagem colaborativa pelas inter-relações pessoais, originando novos aprendizados (SALES; BARROSO; SOARES, 2008).

Entretanto, considerando o universo da aprendizagem virtual, não se pode dissociar deste o processo avaliativo, porém, não sendo suficiente defini-lo quantitativamente, deve-se também discernir imperfeições qualitativamente.

Com base nestas observações, identificou-se a necessidade de agregar ao Fórum P e R (Perguntas e Respostas) do Ambiente Virtual de Aprendizagem (AVA) Moodle, um instrumento de avaliação formativa não-linear que valorizasse qualitativamente as postagens efetivadas. Um modelo que promova a autonomia do aluno na construção do seu conhecimento e que possa, a partir de sua primeira contribuição, não ser influenciado pelas mensagens dos demais, promovendo-lhe, assim, maior subjetividade.

Desta forma, depara-se com uma indagação: como desenvolver uma metodologia de avaliação e inserir modificações de configurações na ferramenta assíncrona Fórum P e R, no intuito de possibilitar o aprendizado, onde colaboração e interação contribuam para a autonomia do aprendiz?

Logo, configura-se nesta pesquisa um cenário que permita a facilitação e praticidade do gerenciamento do processo avaliativo de caráter quali-quantitativo.

Assim, o objetivo deste trabalho é conceber um modelo formativo, não-linear de avaliação, aplicado ao Fórum P e R do AVA Moodle, denominando-o Fórum P\&R LV, como uma contribuição ao Modelo LV e seus Vetores-Aprendizagem em EaD on-line (SALES, 2010).

O Modelo LV (Learning Vectors) são representações vetoriais criados para auxiliar o processo avaliativo em AVA, pois como recurso virtual, o mesmo elucida o caráter quantitativo e qualitativo quanto a performance do discente, facilita um frequente feedback como também não sobrecarrega o docente (SALES; BARROSO; SOARES, 2008).

Neste prisma vetorial, faz-se essencial propor uma especificação matemática e mecanismos de avaliação baseados na não-lineariedade, avaliando seu comportamento em uma situação real de uso em um curso on-line em funcionamento.

Este trabalho está organizado nas seguintes seções: a seguir trata-se de modelos pedagógicos relacionados à avaliação em $\mathrm{EaD}$ on-line; na segunda seção abordam-se os percursos metodológicos empregados; na terceira seção é apresentada uma pesquisa de satisfação e discutem-se seus resultados e, por fim, apresentam-se as considerações finais.

\section{MODELOS PEDAGÓGICO EM AVALIAÇÃO EM EaD ON-LINE}

No cenário educativo perdura a visão Sócio-Construtivista de Vygotsky (1984), na qual o sujeito responde aos estímulos externos provocados pelas relações interpessoais deste com o meio. Além disto, nesta concepção o aluno é levado a descobrir conteúdos, analisar soluções e tomar decisões, apresentando-se como sujeito ativo, reflexivo e construtor participativo, demonstrando maior autonomia.

Assim, permite-se que o aprendiz faça uso dessa autonomia, como fator motivador, perceptível de mudanças comportamentais, que favoreçam a autoaprendizagem; na ação de um ser ativo, interativo, possa perceber-se como ser potencial e consciente de seus atos, tornando-se agente responsável por seu próprio desenvolvimento, como parte de um contexto maior, o da aprendizagem. 
Logo, o sujeito aprende com base no desempenho das suas próprias ações/interações com o meio social, buscando potencializá-las; dessa forma, aproximase da Zona de Desenvolvimento Proximal (ZDP), definida por:

Distância entre o nível de desenvolvimento real, que se costuma determinar através da solução independente de problemas, e o nível de desenvolvimento potencial, determinado através da solução de problemas sob a orientação de um adulto ou em colaboração com companheiros mais capazes. (VYGOTSKY, 1984, apud OLIVEIRA, 1997, p. 60).

Ciente da importância de atingir o nível potencial, investindo na colaboração com companheiros mais capazes, opta-se pelo apoio ao método proposto por Mazur et al., (2007) denominado Peer Instruction (PI), ou Aprendizagem por Pares, defende a construção coletiva do conhecimento através do diálogo entre os sujeitos.

Esta, por sua vez, é considerada uma metodologia ativa. Suas premissas cultivam o eixo do processo colaborativo - um modelo que infere o diálogo entre sujeitos, visando à construção cooperativa do conhecimento, promovendo uma aprendizagem ativa, ou seja, formada por sujeitos ativos (TOPPING, 2007). Logo, sobre o mesmo modelo, postula Caldwell $(2007$, p.18) que:

A aprendizagem por pares (também conhecida na literatura como instrução por pares ou PI) pressupõe o pensamento e as respostas individuais do aluno, independentes e, em seguida, em grupos, busque alcançar uma resposta consensual em seus grupos (tradução nossa).

Logo, a interação é o centro deste processo, exaltando o modelo dialógico. Frisase, dessa forma, a magnitude das interações que proporcionam construções colaborativas que remodelam o contexto educacional, apoiado pelo uso da tecnologia, como a disponibilidade e combinação de seus recursos. Assim, o uso destes nos ambientes virtuais favorece o processo de ensino e aprendizagem.

Nesse contexto pode-se aplicar a utilização de AVA na aproximação de pessoas com níveis semelhantes de desenvolvimento, ou o par mais capaz. A proposta é constituir zonas que permitam potencializar a construção e reconstrução do conhecimento. Em outras palavras, aprender ensinando.

Para que se efetive, é necessário o uso das tecnologias suportando o modelo de $\mathrm{EaD}$, assim como é essencial a lente da avaliação neste processo. Segundo Zabala (1998, p. 200):

[...] a finalidade da avaliação é ser um instrumento educativo que informa e faz uma valoração do processo de aprendizagem seguido pelo aluno, com o objetivo de oportunizar, em todo momento, as propostas educacionais mais adequadas.

Contudo, vale ressaltar que o desenho e a finalidade da avaliação visam apoiar a melhoria do processo. Assim, como uma fuga ao processo tradicional de avaliação, aponta-se a avaliação formativa, que é reguladora, motivadora e orientadora. Para Perrenoud (1999, p.103) "é formativa toda avaliação que ajuda o aluno a aprender e a se desenvolver, ou melhor, que participa da regulação das aprendizagens e do desenvolvimento no sentido de um projeto educativo".

Entretanto, este modelo avaliativo destina-se identificar deficiências em aprendizagens, essenciais à abertura de outros caminhos que oportunizem novas 
situações de aprendizagens. Logo, a observação de modelos avaliativos não lineares apresenta-se como resposta na valorização da subjetividade.

Como assinala Araújo (2004, p.50), "modelos lineares não são suficientes para capturar toda a interação que é necessária e que acontece nas comunidades virtuais". Diante da subjetividade nas inter-relações em AVA, a avaliação precisa estar apoiada em teorias de sistemas dinâmicos não-lineares.

Sales (2010), em Learning Vectors (LV): um modelo de avaliação da aprendizagem em EaD online aplicando métricas não-lineares, apresenta um modelo avaliativo que ressalta a importância não tão somente dos fatores quantitativos, mas ressalta os qualitativos, de aprendizagem.

Este, intitulado Modelo LV, permite mensurar as interações colaborativas para avaliação de desempenho. Contudo, o autor desenvolveu um instrumento de avaliação quali-quantitativo para mediar o processo de ensino e aprendizagem, e um mecanismo semiautomatizado para o controle acadêmico.

\section{PROCEDIMENTOS METODOLÓGICOS}

O LMS Moodle - Modular Object-Oriented Dynamic Learning Environment - é um sistema de gestão do ensino e da aprendizagem que visa gerenciar a aprendizagem sob uma perspectiva colaborativa, disponível para o suporte a cursos on-line. Ambiente que abrange ferramentas, tanto síncronas quanto assíncronas, tais como: fórum, chat, tarefa, quiz, wiki, dentre outras.

Em observância aos recursos existentes, como Chat $L V$, Wiki $L V$, visando potencializar a comunicação centrada na interação dos envolvidos, optou-se por uma investigação ao fórum P e R.

O mesmo, oculta as contribuições dos demais alunos, até que a sua primeira seja efetivada, assim as demais se tornam visíveis (Figura 1). O objetivo é prover igual oportunidade aos alunos, incentivando-os ao raciocínio autônomo e, por conseguinte, uma discussão contextualizada e a construção consensual de forma colaborativa.

Figura 1 - Modelo de postagem oculta

\begin{tabular}{|c|c|}
\hline $\begin{array}{l}\text { Assunto (oculto) } \\
\text { Autor (oculto) } \\
\text { Você não pode ver esta mens }\end{array}$ & ão publicou nada nesta discussão. \\
\hline$\left\{\begin{array}{l}\text { Re: Avaliação em EAD } \\
\text { por aluno } 4 \text { - Friday, } 26\end{array}\right.$ & \\
\hline \multicolumn{2}{|c|}{ Contribuição do Aluno Teste 4 no FórumPR sobre Avaliação em EAD. } \\
\hline Média das avaliações: - & Mostrar principal | Editar | Excluir | Responder \\
\hline
\end{tabular}

Fonte: Help Class Online

Assim, pela ausência de um modelo de avaliação não-linear no fórum $\mathrm{P}$ e R, utilizou-se do Modelo LV (SALES, 2010) sob a metodologia PI, como, uma nova especificação matemática, concebendo, assim, o Fórum P\&R LV, explanado a seguir.

No Modelo LV as notas relativas às postagens realizadas no Fórum P\&R LV são representadas, numa escala de 0 a 10, pelo Vetor-Aprendizagem (Figura 2a e 2b) - um instrumento de mensuração vetorial representativo do rendimento do aluno. Na Figura 
2c, tem-se a simbologia gráfica vetorial, apresentando tanto as componentes envolvidas, $\mathrm{LV}_{\mathrm{x}}$ e $\mathrm{LV}_{\mathrm{y}}$, o tamanho do vetor, quanto o deslocamento angular deste.

\section{Figura 2 - Vetor-Aprendizagem e sua representação gráfica}

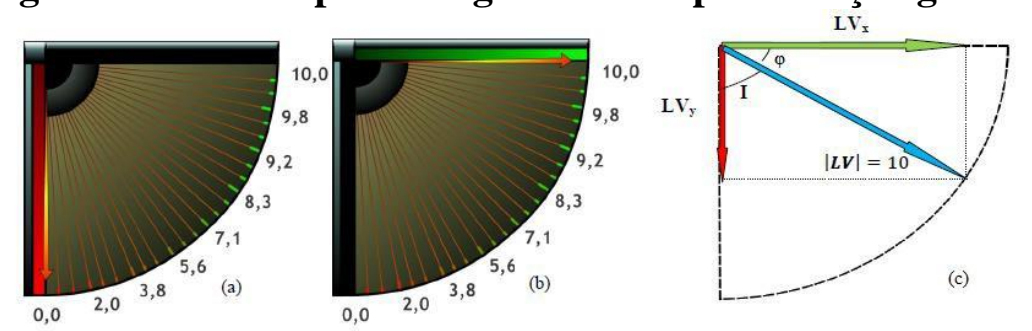

Fonte: Sales (2010).

Já o professor/tutor faz uso dos LV Ícones para efetivar cada postagem dos alunos, sendo estes, computacionalmente representados por uma escala numérica variando de 0 (zero) a 4 (quatro), denominada Coeficiente de Passo (CP), associados à diferentes menções qualitativas (Tabela 1).

Tabela 1 - Menções Qualitativas x LV Ícones

\begin{tabular}{|c|c|c|c|c|c|c|}
\hline LV Ícones & $\odot$ & $\odot$ & $\odot$ & $\odot$ & $\Theta$ & $\Theta$ \\
\hline Menções & Muito Bom & Bom & Regular & Fraco & Não Satisfatório & Neutro \\
\hline
\end{tabular}

Fonte: Sales (2010).

A escolha do LV Ícone influencia no deslocamento do vetor, representado a partir do $4^{\circ}$ quadrante, que inicialmente assume valor $\varphi=-90^{\circ}$ (nota mínima - Figura 2a) e desloca-se até, no máximo, $\varphi=0^{\circ}$ (nota máxima - Figura $2 b$ ). Sob uma variação angular denominada passo padrão, fixada em $=3,75^{\circ}$, as notas, denominadas componente horizontal, são calculadas utilizando-se a seguinte equação:

$$
\left.\mathrm{LV}_{\mathrm{x}}=10^{*} \cos \left[\left(-90^{\circ}\right)+\mathrm{I}_{\mathrm{n}}\right)\right]
$$

$\begin{array}{llll}\text { Com: } & & \text { e } 3,75^{\circ} & \\ & & & \\ \text { Sendo: } & \{ & 3 & \\ \{ & 5 & & \end{array}$

Esta necessita do somatório das $\mathrm{n}$ interações no fórum, representado por $I_{n}$. Este, por sua vez, conta com o coeficiente do passo (CP), a variação angular ( ) e o fator multiplicador $(\mathrm{m})$, o qual confere diferentes pesos a diferentes mensagens, cronologicamente inseridas (Tabela 2) e foi escolhido com base nas configurações dispostas no Modelo LV. Neste caso, o Fórum P\&R LV foi configurado para um número mínimo de 4 mensagens, acordado por meio de Contrato Didático.

Tabela 2-Fator multiplicador (m) x Coeficiente do Passo (CP)

\begin{tabular}{|c|c|c|c|c|c|}
\hline $\begin{array}{l}m \\
\mathrm{CP}\end{array}$ & $\stackrel{4}{\ominus}$ & $\stackrel{3}{\odot}$ & $\stackrel{2}{\odot}$ & $\stackrel{1}{\odot}$ & $\Theta^{0}$ \\
\hline 1 & 4 & 3 & 2 & 1 & 0 \\
\hline 2 & 8 & 6 & 4 & 2 & 0 \\
\hline
\end{tabular}

Fonte: Sales (2010). 
As duas primeiras usando fator multiplicador $m=2 \mathrm{e}$, as duas outras, com fator multiplicador $m=1$, perfazendo o necessário para que, desde que avaliadas com o LV Ícone Azul, $(\mathrm{CP}=4)$, o aluno possa atingir a nota máxima (Tabela 3).

Tabela 3 - Número Mínimo de Mensagens

\begin{tabular}{|c|c|c|c|c|c|c|}
\hline \multicolumn{2}{|c|}{$\begin{array}{c}\text { Coeficiente do Passo } \\
(\boldsymbol{C P})\end{array}$} & $\mathbf{4}$ & $\mathbf{3}$ & $\mathbf{2}$ & $\mathbf{1}$ & $\mathbf{0}$ \\
\hline \multirow{4}{*}{$\begin{array}{c}\text { Ordem da } \\
\text { Mensagem/ } \\
\text { Nota }\end{array}$} & $\mathrm{I}_{1}$ & $8 \varphi / 5,00$ & $6 \varphi / 3,83$ & $4 \varphi / 2,59$ & $2 \varphi / 1,31$ & $0 \varphi / 0,00$ \\
\cline { 2 - 7 } & $\mathrm{I}_{2}$ & $8 \varphi / 8,66$ & $6 \varphi / 7,07$ & $4 \varphi / 5,00$ & $2 \varphi / 2,59$ & $0 \varphi / 0,00$ \\
\cline { 2 - 7 } & $\mathrm{I}_{3}$ & $4 \varphi / 9,66$ & $3 \varphi / 8,31$ & $2 \varphi / 6,09$ & $1 \varphi / 1,31$ & $0 \varphi / 0,00$ \\
\cline { 2 - 7 } & $\mathrm{I}_{4}$ & $4 \varphi / 10,00$ & $3 \varphi / 9,24$ & $2 \varphi / 7,07$ & $2 \varphi / 1,31$ & $0 \varphi / 0,00$ \\
\cline { 2 - 7 } & $\mathrm{I}_{\mathrm{n} \geq 5}$ & $+1 \varphi$ & $+1 \varphi$ & $+1 \varphi$ & $-1 \varphi$ & $-1 \varphi$ \\
\hline
\end{tabular}

Fonte: Adaptada de Sales (2010).

As mensagens seguem os seguintes critérios: $\left(\mathrm{I}_{1}\right)$ : Mensagem Principal: compreensão acerca do tema proposto; $\left(\mathrm{I}_{2}\right)$ : $1^{\mathrm{a}}$ Mensagem de Interação: resposta, comentário, sugestão, crítica ou concordância, seguida de justificativa, a uma mensagem já realizada; e, $\left(\mathrm{I}_{3}\right.$ e $\left.\mathrm{I}_{4}\right)$ : demais mensagens de interação.

Observa-se que, a partir da quinta mensagem, $\left(\mathrm{I}_{\mathrm{n} \geq 5}\right)$, temos tanto um fator motivador, associando-se uma variação angular $\Delta=+1 \varphi$, caso a interação seja classificada com os LV Ícones Amarelo, Verde ou Azul, respectivamente: $\mathrm{CP}=2, \mathrm{CP}=3$ ou $\mathrm{CP}=4$, quanto um fator penalizador, com variação angular $\Delta=-1 \varphi$, caso sejam o Vermelho ou Laranja, ou, $\mathrm{CP}=0$ e $\mathrm{CP}=1$.

Observam-se no Vetor-Aprendizagem (Figura 2c), os componentes horizontais e verticais, representando a positividade e a negatividade de desempenho de um aluno, respectivamente.

Já que, na demonstração gráfica do vetor, visualiza-se um triângulo retângulo e tendo-se, inicialmente já definida a componente horizontal, ou $\mathrm{LV}_{\mathrm{x}}$, equaciona-se o valor do componente vertical, ou $\mathrm{LV}_{\mathrm{y}}$, aplicando-se o Teorema de Pitágoras:

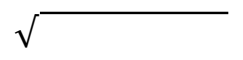

Com os dois componentes do vetor definidos, ou seja, $\mathrm{LV}_{\mathrm{x}}$ (Positividade) e $\mathrm{LV}_{\mathrm{y}}$ (Negatividade), usa-se a Equação a seguir, para encontrar o valor equivalente ao Fator $\beta$, que é a relação Positividade/Negatividade (P/N) do Modelo LV pressuposto pelos autores Losada e Heaphy (2004) para identificar o nível de conectividade entre os membros de um mesmo grupo. Dessa forma, as interações são mensuradas matematicamente pela relação das taxas de positividade e negatividade, juntamente aos pesos atribuídos às avaliações, resultando no desempenho daquele grupo.

Sendo: $\beta=$ Fator beta, $\mathrm{P}=$ Positividade, $\mathrm{N}=$ Negatividade, $* \mathrm{LV}=$ Quantidade de LV Ícones referentes a cada cor e NTF = Número Total de Faltas. (Na proposta foi considerado $\mathrm{NTF}=0$, pela presença dos alunos à atividade). 
Calculado o Fator $\beta$, observa-se pelo resultado que podemos enquadrá-lo de acordo com a Escala de Desempenho do Fator (Tabela 2) para identificarmos o nível deste, assim, conferindo o grau de conectividade daqueles envolvidos.

Tabela4 - Escala de Desempenho do Fator $\beta$

\begin{tabular}{|c|c|c|c|c|c|}
\hline $\begin{array}{c}\text { Escala de } \\
\text { Desempenho }\end{array}$ & Muito Alto & Alto & Médio & Baixo & $\begin{array}{c}\text { Muito } \\
\text { Baixo }\end{array}$ \\
\hline Fator $\beta$ & $\beta \geq 3,78$ & $2,62 \leq \beta<3,78$ & $0,90<\beta<2,62$ & $0,30 \leq \beta<0,90$ & $0 \leq \beta<0,30$ \\
\hline
\end{tabular}

Fonte: Sales (2010).

Pelo detalhamento da tabela, observa-se que o fator $\beta$ pode ser utilizado para diferenciar alunos com notas finais iguais ou próximas, expressando quantitativamente o desempenho destes.

Portanto, ao longo da aplicação, percebeu-se que o diálogo mediado pelo Modelo LV utilizada, possibilita uma facilitação na regulação da aprendizagem seguindo um modelo avaliativo formativo. Assim, facilita ao mesmo tempo em que propicia o desenvolvimento, através de processos interativos entre os atores existentes.

Um estudo de caso, baseado na metodologia citada, foi aplicado no intuito de testar a ferramenta modelada.

\section{APRESENTAÇÃO E DISCUSSÃO DOS RESULTADOS}

Os dados adquiridos através de questionário de satisfação, analisados e aqui representados têm por base um estudo de caso realizado na disciplina de Avaliação Educacional em EaD, junto ao Curso de Especialização em Educação a Distância da Universidade Federal do Ceará (UFC), totalizando um público-alvo de 76 (setenta e seis) alunos inscritos, dos quais 64 acessaram efetivamente o Fórum P\&R LV. Vale salientar que a maior parte dos alunos traz consigo um bom domínio diante das Tecnologias Digitais de Informação e Comunicação (TDIC).

De acordo com a opinião da maioria dos alunos, ressalte-se que, para uma troca frequente, intensa e aprofundada, o número de atores envolvidos irá influir no resultado. Uma grande dificuldade encontrada quanto da realização do processo avaliativo foi o número excessivo de alunos ativos.

Observou-se ainda que $98 \%$ acordaram quanto o Fórum P\&R LV promover uma construção interativa e uma aprendizagem colaborativa, sobretudo, significativa. Portanto, visando essa construção, o feedback constante é parte regulador do processo de aprendizagem e assim reconhecido pelos usuários. Salienta-se que, a totalidade dos participantes o aprovou como instrumento usado para reduzir o distanciamento físico e, também, como um instrumento que mensura o desempenho dos alunos - uma modelo para alimentar o processo avaliativo formativo.

\section{Gráfico 1 - O Fórum P\&R LV de discussão oportuniza um processo de construção interativo e colaborativo?}




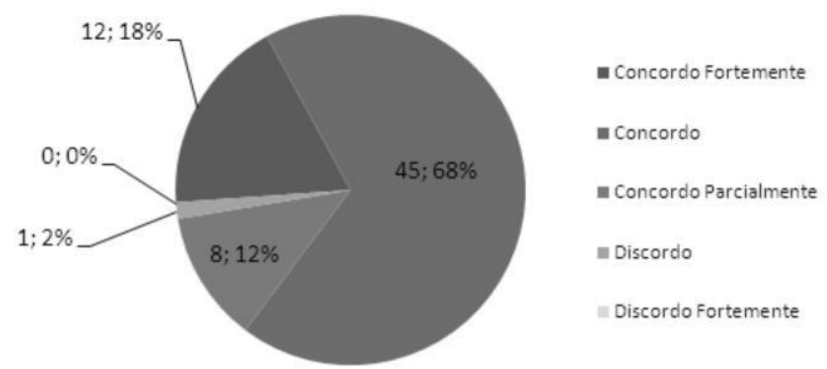

Fonte: Elaborado pelos autores

Logo, vale destacar que uma grande parte, ou 93\%, concorda que o modelo aplicado possa promover uma maior reflexão crítica individualizada, e, por conseguinte, observando-se os direcionamentos através do feedback regulador do Professor/Tutor, visando prover um formato interativo de aprendizagem colaborativa, que assegure igual acesso, raciocínio e produção, sobretudo, uma maior autonomia.

\section{Gráfico 2 - O Fórum P\&R promove uma reflexão crítica individualizada?}

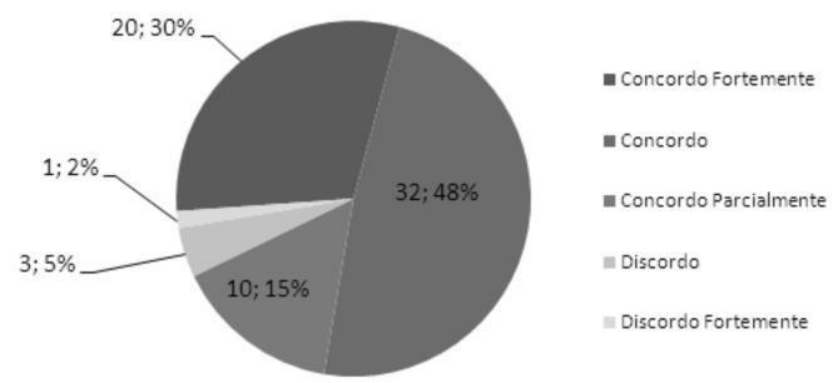

Fonte: Elaborado pelos autores

Assim, para sustentar o modelo formativo de avaliação, tivemos atrelados às menções qualitativas, os LV Ícones, como instrumentos iconográficos, capazes de possibilitar uma comunicação/avaliação objetiva, de forma efetiva.

Tal comunicação construída no AVA, através das efetivas interações entre seus atores, leva a uma dimensão diferenciada, onde permite o aluno sair de sua zona de conforto, superar desafios e, intensamente, mergulhar em um novo plano, o que poderá contribuir para uma aprendizagem eficaz, guiada por um modelo formativo não-linear que considera a subjetividade.

Subjetividade esta, capturada por menções qualitativas descritas no Modelo LV de avaliação formativa não-linear, a saber: Muito Bom, Bom, Regular, Fraco, Não Satisfatório, e Neutro, associadas às imagens de uma linguagem iconográfica, tornam-se mecanismos de representação da avaliação por parte do Professor/Tutor, em que foram interpretadas positivamente pelos alunos, num total de $94 \%$ dos envolvidos.

\section{Gráfico 3 - As menções qualitativas refletem claramente a análise do Professor/Tutor?}

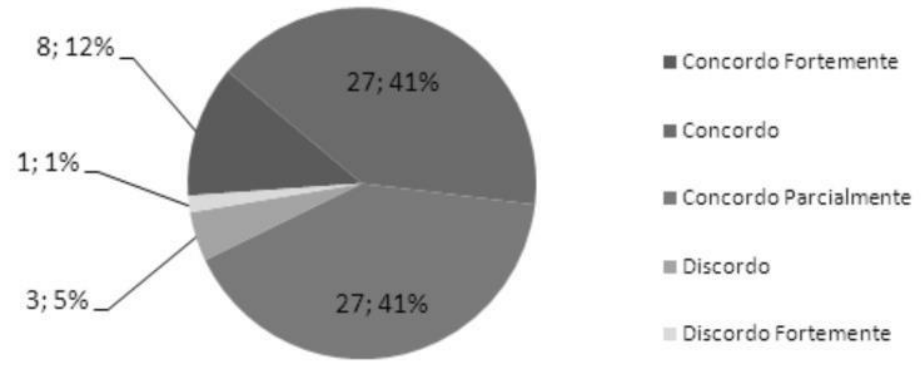


Contudo, vale ressaltar inclusive, que houve uma plena aceitação da modelagem aplicada, visto que a grande maioria, ou 95\%, faria uso do modelo proposto Fórum P\&R LV, como um meio de promover um grande nível de satisfação dentre seus usuários.

\section{Gráfico 4 - Em sua prática de ensino, você faria uso do Fórum P\&R LV?}

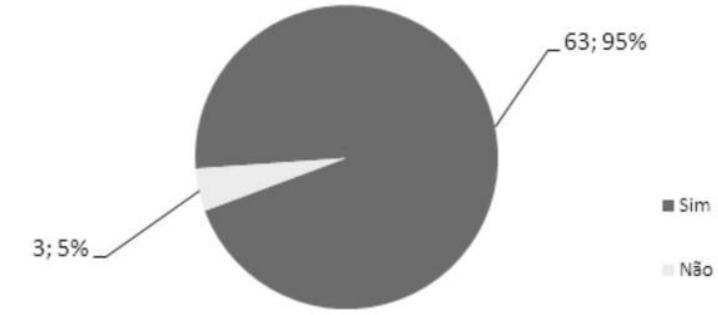

Fonte: Elaborado pelos autores

\section{CONSIDERAÇÕES FINAIS}

Buscou-se, neste trabalho, uma proposta educativa em EaD para subsidiar um processo de avaliação quali-quantitativo, logo, aplicou-se o Modelo LV (SALES, 2010) ao Fórum $\mathrm{P}$ e R, por sua especificidade.

A concepção do Fórum P\&R LV (Fórum Perguntas e Respostas Learning Vectors) propõe um instrumento não-linear de avaliação formativa, sob uma nova especificação matemática, com metade do valor de passo padrão inicialmente proposta por Sales (2010), permitindo uma reconfiguração do número de mensagens mínimas necessárias para atingir nota máxima.

Apoiado pela metodologia ativa de Aprendizagem por Pares, a proposta sob a nova especificação possibilitou um real aprendizado, dissipado entre interação e colaboração. Logo, a aplicação permitiu ao aluno regular a dimensão de sua própria aprendizagem.

Os resultados do estudo de caso apontaram que o Fórum P\&R LV promoveu construções colaborativas com valorização da subjetividade no diálogo efetivado. Inicialmente, um modelo sob um prisma individual que, consequentemente, cedeu espaço ao impessoal, despontando ao modelo colaborativo de aprendizagem e, assim, rompendo com os moldes tradicionais de métricas lineares de avaliação, permitindo uma mensuração de caráter quali-quantitativo de forma justa, afetiva e motivadora.

Desse modo, constatou-se no modelo aplicado uma contribuição para o modelo formativo, tendo verificado a pertinência da avaliação formativa em conjunto a um modelo não-linear com mediação iconográfica por como um processo regulador e contínuo de aprendizagem.

Conclui-se que, a concepção de um mecanismo computacional de avaliação formativa não-linear, de caráter quali-quantitativo, objeto desta pesquisa, permitiu as mais variadas relações entre os envolvidos, promovendo-os. Ao mesmo tempo, respeitando a individualidade, subjetividade e colaboração, além da consequente aprendizagem pelas instruções expostas.

Por fim, o Fórum P\&R LV permitiu conferir resultados amplamente satisfatórios como um modelo não-linear de avaliação com suporte do Modelo LV e seus VetoresAprendizagem. 


\section{REFERÊNCIAS}

ARAÚJO, L. H. L. Uma Aplicação da Dinâmica Não-linear para Avaliação de Desempenho de Comunidades Virtuais de Aprendizagem: além da tela do computador: linguagem, emocionalidade e corporalidade. Dissertação de Mestrado em Gestão do Conhecimento e Tecnologia da Informação. Brasília: UCB, 194p, 2004.

CALDWELL, J. E. Clickers in the Large Classroom: current research and Best practice tips. CBE Life Sci. Educ. 6, 9 -20, 2007.

LOSADA, M.; HEAPHY, E. The Role of Positivity and Connectivity in the Performance of Business Teams: a nonlinear dynamics model. American Behavioral Scientist, vol. 47, $\mathrm{n}^{\circ}$ 6, 740-765, 2004.

MAZUR, Eric. CROUCH, Catherine H. WATKINS, Jessica. FAGEN, Adam P..Peer Instruction: engaging students one-on-one, all at once, in Reviews in Physics Education Research, Ed. E.F. Redishand P. Cooney, pp. 1 -1 (American Association of Physics Teachers, College Park, MD), 2007.

MOORE, Michael G.; KEARSLEY, G. Educação a Distância: uma visão integrada. Tradução Roberto Galman. - São Paulo: Thomson Learning, 2007.

OLIVEIRA, Marta Khol de. Vygotsky: aprendizado e desenvolvimento: um processo sócio-histórico. São Paulo: Scipione, 1997.

PERRENOUD, P. Avaliação: da excelência à regulação das aprendizagens entre duas lógicas. Porto Alegre: Artmed, 1997.

SALES, G. L. Learning Vectors (LV): um modelo de avaliação da aprendizagem em EaD online aplicando métricas não-lineares / Gilvandenys Leite Sales. - 2010. 236f. : il.; color. ; enc. Tese (Doutorado) - Universidade Federal do Ceará, Curso de Pós Graduação em Engenharia de Teleinformática, 2010.

SALES, G. L.; BARROSO, G. C.; SOARES, J. M. Learning Vectors (LVs) um Instrumento Automatizado de Avaliação para Suporte a Aprendizagem em EaD. In: RENOTE Revista Novas Tecnologias na Educação. Julho 2008 V6 N1. CINTED/UFRGS Porto Alegre-RS.

TOPPING, K. Peer-Assisted Learning. In N. Salkind (Ed.), Encyclopedia of Educational Psychology. Thousand Oaks, CA: Sage Pub, 2007.

VYGOTSKY, L. S. Pensamento e Linguagem. São Paulo: Martins Fontes, 1984.

ZABAlA, A. A. Prática Educativa: como ensinar. Trad. Ernani F. da F. Rosa. Porto Alegre: Artmed Editora, 1998. 